burns, dehydration, prior IV chemotherapy, and IV drug use make peripheral veins difficult to identify and cannulate.

Critical time can be lost while attempting to obtain vascular access, potentially increasing patient morbidity and mortality. On the other hand, central venous access is time-consuming and poses complication risks like infections and pneumothorax.

In these instances, Intra-osseous (IO) contrast injections are beneficial to obtain good quality trauma scans for patients from the Emergency Department (ED).

Methods Multi-professional stakeholders (Radiologists, ED consultants, and Radiographers) met to identify the need for IO service in Aberdeen Royal Infirmary. This was followed by discussions at the CT user's group meeting. A need for developing a local protocol for IO contrast service was identified for patients with no IV access. A draft protocol was created and then modified to meet local needs.

Interventions Once the protocol was finalised, IO contrast service was introduced for trauma patients without IV access. The local protocol gives clear information about placement and checking of IO needle, local anaesthetic administration and the technique for contrast administration.

Results Since implementing the protocol, 3 successful trauma scans have been performed using IO access. The quality of images has been good with no recorded complications.

Conclusions IO contrast provides an effective and safe alternative to IV access. Good quality images can be obtained using established protocols.

Presenting author Dr Yajur Narang*

\section{IS THERE A NEED TO CHANGE THE DELIVERY OF LEADERSHIP TRAINING TO OUR FUTURE DOCTORS?}

Jabin Chowdhury, Victor Kimani, Haroon Ali Shah. UK

10.1136/leader-2021-FMLM.37

The General Medical Council (GMC) has advocated for wider leadership and management responsibilities for doctors and the Birmingham Medical Leadership Society (BMLS) aims to address ways in which students and doctors can be supported through this. Collecting data on this topic is pivotal to improving the way in which medical leadership is taught to prepare students to take on leadership roles.

The survey conducted addressed the importance of leadership training. After analysing 122 responses, the consensus is that medical leadership training is important with $61.5 \%$ of people rating it 5 on the Likert scale. Participants were given the opportunity to rate how they thought medical education would best be learned. $77.9 \%$ agreed an NHS paired learning scheme where students are paired up with a practising healthcare professional, would be a suitable method. Teaching from and shadowing of NHS managers proved popular, as did leadership workshops. The lack of representation of foundation doctors and those further on in their training has created a need for more data from doctors of different grades, to see if their views on medical leadership significantly differ.

Should leadership teaching be introduced earlier on in medical school through the methods

BMLS anticipates that earlier and better medical leadership education will make more confident doctors with capacity to take on leadership roles. Shifting the leaders of healthcare services towards those with clinical background prioritises patients at the centre of care.

Using student views to tailor medical education feels productive to producing the next generation of doctors who are better equipped with skills required to take on leadership roles. BMLS hopes medical schools will listen to these views and take action and that medical leadership training is emphasised in Good Medical Practice. This serves our healthcare service which in turn makes for better quality and safety of care.

\section{LEADERSHIP IN THE MANAGEMENT OF PATIENTS WITH MULTIPLE CONDITIONS: AN IMPACT EVALUATION OF BMJ BEST PRACTICE COMORBIDITIES}

Kieran Walsh. BMJ Knowledge Centre, BMJ, UK

\subsection{6/leader-2021-FMLM.38}

BMJ Best Practice Comorbidities has been created by the BMJ Knowledge Centre. This work was carried out by BMJ staff and was done in collaboration with healthcare professionals in training in the UK. One in three adults admitted to hospital in the UK have five or more conditions. People with comorbidities have poorer functional status, quality of life, and health outcomes, and are higher users of ambulatory and inpatient care than those without comorbidities. This poses a problem for patients, healthcare professionals, health leaders, and health systems. In light of this, BMJ Best Practice launched a new tool - BMJ Best Practice Comorbidities. This tool enables healthcare professionals to access detailed guidance on how to manage patients with multiple conditions and thus provide holistic care. In a pilot evaluation, we made this tool available to a group of healthcare professionals in training. We then asked them to fill in a simple questionnaire outlining what difference, if any, the tool made to their practice. We measured the effect of any improvement as a result of using the tool by means of a short questionnaire. We asked the healthcare professionals to describe the circumstances in which they had used the tool, whether the tool made any difference to their practice, and what impact this had on patient care. The evaluation showed that BMJ Best Practice Comorbidities is effective at helping healthcare professionals to improve the care that they provide to patients with multiple conditions (the resource helped users to improve practice in $70 \%$ of cases). When it doesn't change practice, it can still have an effect by reassuring users that their practice is correct (this occurred in $15 \%$ of cases). This evaluation showed impact in improving the practice of healthcare professionals in delivering high-quality and safe care for patients with comorbidities.

\section{LOCAL TRAINING PROGRAMME FOR ANAESTHETISTS AND FUTURE LEADERS}

Gregory Fraser, Emma James. Imperial College Healthcare Trust, Royal National Orthopaedic Hospital NHS Trust

10.1136/leader-2021-FMLM.39

Primary issues - centralised training programme by HEE for anaesthetics, limited number of training posts compared to 
applicants. Local anaesthetic training programme being set up via the CESR pathway to help train more anaesthetists. Funded locally by 2 separate NHS trusts

Secondary issues - largest elective waiting list ever in NHS needs more staff trained in surgical specialties. Multiple recruitment rounds for trusts locally to fill non training posts. Wasted time for trainees and HR spent on induction and mandatory statutory training when having to rotate so regularly.

Two Healthcare trusts have collaborated to provide a local training pathway. Previous issues with CESR applications have been the difficult in collecting and providing retrospective evidence of training. By designing a training programme which follows the structure of the GMC guidance for CESR anaesthetic pathway, song with the RCoA curriculum - the typical issues with CESR applications can be avoided by collecting evidence prospectively. The relevant organisations and individuals collaborated through networking and planning towards a shared goal of providing a training programme for future anaesthetists.

This programme provides an opportunity for future leadership development. Leadership is compulsory in traditional training programmes but not necessarily formally taught. By building an autonomous curriculum to match the GMC guidance, leadership and understanding of NHS management will be introduced from the beginning of the programme, and active leadership positions within the trust will be encouraged.

The programme will run over four years across five hospital sites. The time spent at each site will be based upon the trainee's preferences, training needs and service provision. Across all sites there will be a collaborative approach to quality improvement and audits, to maximise output which both the individuals and organisations will benefit

\section{MANAGING MEDICAL EDUCATION DURING THE PANDEMIC-EXPERIENCE OF A PRIVATE MEDICAL SCHOOL IN MALAYSIA}

${ }^{1}$ Ganesh Ramachandran, ${ }^{2}$ Aung Ko Ko Min, ${ }^{3}$ Munandy Alagar. 'Taylor's University Malaysia; ${ }^{2}$ MAHSA University Malaysia; ${ }^{3}$ UCSI University Malaysia

\subsection{6/leader-2021-FMLM.40}

This work was undertaken in a private medical school in Malaysia, it looks at interventions undertaken due to the COVID-19 pandemic and impact on students. The pandemic disrupted the accepted norms for delivery in the faculty, requiring quick changes to ensure uninterrupted teaching, learning and assessment for students. The problems were instant conversion to on online format with the use of simulation and recorded practical sessions for theory and practical sessions respectively. The other major issue was to educate staff and students on this matter and update delivery platforms.

The immediate solutions

- Use the platforms immediately available.

- Determine a minimum level of competency required to deliver the curriculum.

- Engage stakeholders.

- Ensure faculty development

- Adhere to regulatory frameworks.
This was communicated to all stakeholders and lines of communication formed to engage and mentor students through the period. Feedback on the sessions were sought. Students were constantly reminded this was non-punitive. Objective measures were to compare assessment outcomes of high stakes examinations from the previous years. Questionnaire surveys were used for feedback. Pass rates with previous cohorts were compared. The preliminary result indicates general satisfaction with teaching and learning, preference of the online platform for formative assessment and a comparable performance in all high stakes examinations.

Impact

- The online platform is an acceptable for both instructors and students.

- Formative assessment was preferred on this platform.

- Delivery of the curriculum was continued without disruption if you started with what was available and then developed capabilities

- Communication was important.

Disruption forces innovation and movement out of comfort zones, medical education has largely depended on face-to-face delivery. The pandemic has allowed more innovative delivery.

\section{MAXIMISING ENDOSCOPY TRAINING DURING THE COVID-19 RECOVERY PHASE AT ST MARKS HOSPITAL}

A Shalabi, SM Ha, L Dyall, D Chaterjee, S Pomfret, A Haycock. The Wolfson Unit for Endoscopy, St Mark's Hospital, London, UK

\subsection{6/leader-2021-FMLM.41}

Aims Our unit has 24 gastroenterology and surgical registrars training in endoscopy. Access to endoscopy at St Mark's Hospital has historically been a challenge due to the number of trainees requiring training lists with the demand on the unit to perform tertiary centre complex cases.

The COVID-19 pandemic has further impacted the situation. Trainees have been struggling to meet targets (procedure numbers and exposure to therapy) set out by Joint Advisory Group on GI Endoscopy (JAG) to become competent endoscopists.

Methods Internal teaching courses were run to increase the number of trainers.

An agreement was reached that all lists would be open to trainees without reducing the number of cases. All available endoscopy lists were scrutinised to establish suitability for training. A senior endoscopy trainee acted as the named allocator contacting the other trainees to ascertain their current training needs and weekly commitments. This information was used to allocate appropriate lists to each trainee. A timetable was disseminated to all trainees and trainers every 2 weeks.

The JAG endoscopy training portfolio was used to assess the number of procedures completed by trainees, and a satisfaction survey was conducted pre- and post-intervention.

Results Following our intervention, 10 further trainers were made available. The number of lists attended by trainees increased by 74\%(171 between September 2020 and February 2021 versus 298 between March 2021 and June 2021), gastroscopies performed by 57\% (267 vs 419) and colonoscopies by $111.4 \%$ (175 vs 370$)$. 